\title{
Enantiospecific Adsorption of Cysteine at Chiral Kink Sites on Au(110)-(1×2)
}

\author{
Angelika Kühnle, ${ }^{\ddagger}$ Trolle R. Linderoth, ${ }^{*}$ and Flemming Besenbacher \\ Interdisciplinary Nanoscience Center (iNANO), and Department of Physics and Astronomy, University of Aarhus, \\ DK-8000 Århus C, Denmark
}

Received October 7, 2005; E-mail: trolle@inano.dk

Stereochemistry plays a pronounced role in molecular biology and for the pharmaceutical industry. ${ }^{1}$ An important route to synthesis of enantiomerically pure compounds is enantioselective heterogeneous catalysis, and adsorption of chiral or prochiral molecules onto surfaces has consequently attracted much attention recently. ${ }^{2-17}$ Significant insights have been obtained from studies using the local probe technique of scanning tunneling microscopy (STM): molecular chirality has been directly determined, ${ }^{4,5}$ molecular segregation into homochiral structures ${ }^{4,6-10}$ has been observed, intermolecular chiral recognition ${ }^{11,12}$ has been demonstrated, and chirally specific surface restructuring has been shown to result from adsorption of chiral or prochiral molecules onto surfaces. ${ }^{13-16}$

Terraces of low Miller index single-crystal surfaces are mirror symmetric, and hence achiral, but as pointed out by Gellman et al., kinks at step edges represent chiral centers. ${ }^{18-20}$ Chiral surfaces can therefore be made by cutting metal crystals off high-symmetry directions (see also ref 21). Using averaging techniques such as temperature-programmed desorption, cyclic voltammetry, or Fourier transform infrared reflection-absorption spectroscopy, examples of enantiospecific molecule-substrate interactions on such surfaces have been demonstrated by showing slightly different adsorption energies or spectral features for the different enantiomers of a chiral molecule. ${ }^{19,22-24}$ Insight has also been obtained from theoretical modeling. ${ }^{23,25}$ A direct, microscopic observation of enantiospecific adsorption of chiral molecules onto kink sites has not, however, been reported so far.

In this Communication, we present STM data for the adsorption of the natural amino acid cysteine, $\mathrm{HS}-\mathrm{CH}_{2}-\mathrm{CH}\left(\mathrm{NH}_{2}\right)-\mathrm{COOH}$, onto chiral kink sites on the $(1 \times 2)$ missing-row reconstructed $(110)$ gold surface. ${ }^{26}$ This surface is racemic in the sense that it displays approximately equal numbers of $S$ and $R$ kinks (present as defect sites due to the overall surface roughness), but the local probe character of STM makes it possible to zoom in on specific kink sites, removing the need for specially cut crystals. We find that dimers formed from D/L-cysteine respectively adopt distinctly different adsorption geometries at $S$ kinks, demonstrating enantiospecific adsorption at these chiral centers. Furthermore, dense, homochiral cysteine islands are found to preferentially grow from kink sites of a specific chirality.

The adsorption experiments were performed in an ultrahighvacuum chamber equipped with the home-built Aarhus STM. ${ }^{27}$ The experimental procedure was similar to that described previously. ${ }^{11,28}$ Evaporation of submonolayer coverages of enantiomerically pure D/L-cysteine onto the $\mathrm{Au}(110)-(1 \times 2)$ surface held at room temperature, followed by annealing at $380 \mathrm{~K}$ for $\sim 15 \mathrm{~min}$, leads to a number of coexisting structures on the surface. ${ }^{11,28}$ In particular, the terraces are covered with a dilute phase of chiral cysteine dimers which we have described in detail previously. ${ }^{11}$

¥ Present address: Fachbereich Physik, Universität Osnabrück, Barbarastr. 7 , 49076 Osnabrück, Germany.
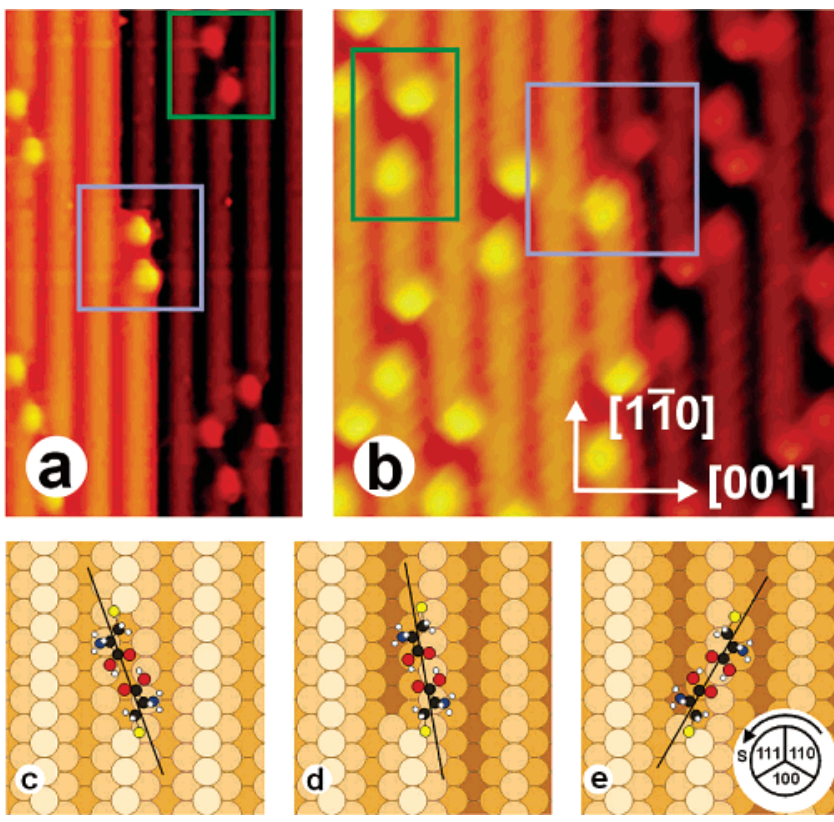

Figure 1. STM images and models for cysteine dimers at $S$ kinks (blue boxes) and at the terrace (green boxes). (a) D-Cysteine dimers. (b) L-Cysteine dimers. (c) Adsorption geometry of a D-cysteine dimer on a four-atomlong vacancy structure, ${ }^{11}$ showing a $20^{\circ}$ rotation compared to the [1-10] direction. (d) Adsorption geometry of a D-cysteine dimer at an $S$ kink site, showing a $10^{\circ}$ rotation. (e) Adsorption geometry of an L-cysteine dimer at an $S$ kink site, showing a $30^{\circ}$ rotation. The assignment of chirality to the kink is based on the indicated ordering of kink microfacets, as introduced in ref 19 .

Depending upon the chirality of the adsorbed cysteine, the main axis through these double-lobed dimers is rotated $20^{\circ}$ either counterclockwise (D-cysteine) or clockwise (L-cysteine) with respect to the [1-10] direction, as illustrated by the dimers in the green boxes in the STM images in Figure 1a,b. The dimers form on fouratom-long vacancy structures in the close-packed gold rows, created by the thermal activation. Theoretical modeling has shown that the adsorption geometry can be understood from a simultaneous optimization of three bonds for each molecule of the dimer, as illustrated in Figure 1c: The sulfur atom is covalently bound at a bridge site next to a low-coordinated gold atom in the topmost row, the nitrogen atom coordinates via a lone pair to the gold surface, and the carboxylic group is forming H-bonds with the carboxylic group of the other molecule in the dimer.

As demonstrated in Figure 1, our current experiments show that the cysteine dimers not only form on the terraces but also adsorb at kink sites. A dimer from D-cysteine adsorbed at an $S$ kink is shown in Figure 1a (blue box). This dimer resembles the dimers adsorbed on the terraces in shape and size; however, it is only rotated about $10^{\circ}$ with respect to the [1-10] direction. A distinctly different situation is found for the L-cysteine dimers adsorbed at 

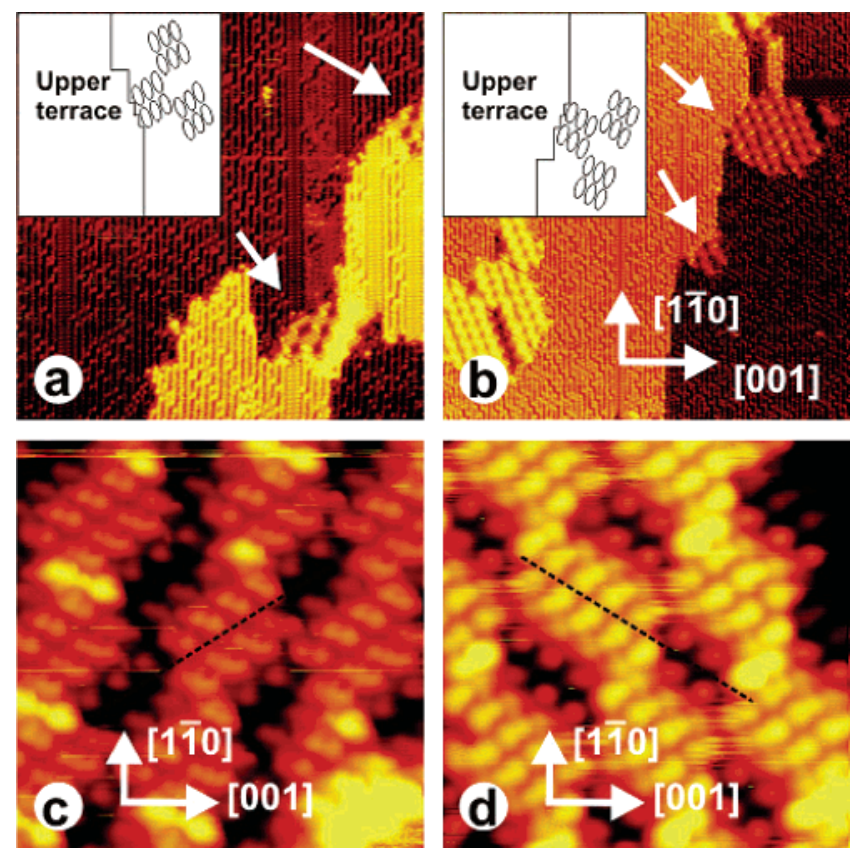

Figure 2. Dense, homochiral cysteine islands growing from chiral kink sites. (a) D-Cysteine islands growing from $S$ kink sites $(449 \AA \times 495 \AA)$. (b) L-Cysteine islands growing from $R$ kink sites $(628 \AA \times 694 \AA)$. (c) High-resolution image of the D-cysteine phase $(70 \AA \times 70 \AA)$. (d) Highresolution image of the L-cysteine phase (70 $\mathrm{A} \times 70 \AA$ ).

an $S$ kink (Figure 1b): In this case, the dimer adsorbed at the kink site is rotated $30^{\circ}$ clockwise, compared to a $20^{\circ}$ clockwise rotation of the L-cysteine dimers adsorbed on the terrace. Since $R$ and $S$ kink sites are mirror symmetric, the adsorption of D/L-cysteine at $R$ kink sites yields the same, but mirror-imaged situation. (A total of 34 cysteine dimers at kinks were analyzed.)

The appearance of cysteine dimers at kinks sites can be understood within the framework established for cysteine dimers at terrace vacancy structures. ${ }^{11}$ We start from the observation that an atomic structure identical to that found at the termination of the vacancies is presented by the kink site. We therefore assume that one of the cysteine molecules adsorbs with the sulfur atom at this site (Figure 1d). The binding of the second sulfur atom in the pair constitutes a change compared to the situation at the vacancy: To achieve adsorption at a bridge site on the lower terrace, the sulfur atom has to move by half a unit cell in the [001] direction, leading to a smaller rotation angle for the D-cysteine dimer at the kink as compared to the situation on the terrace. For L-cysteine dimers at $S$ kink sites, the situation is illustrated in Figure 1e. Again, the binding of the sulfur atom to the upper terrace is identical to the situation at the vacancy, but now the pair has to increase its rotation angle in order to reach the optimum bridge adsorption position on the lower terrace.

The observation of different adsorption geometries at kink sites for different enantiomers does not necessarily imply a preference for adsorption at a given chiral site. However, preferential interaction is seen for a dense cysteine phase, coexisting with the molecular pairs, as shown in Figure 2. A zoom into the dense phase is shown in Figure 2c,d, revealing a high-symmetry direction, which is rotated $55^{\circ}$ clockwise (counterclockwise) with respect to the [1-10] direction for D-cysteine (L-cysteine), as shown by the black dashed lines. The opposite rotation angles for the D/L-cysteine demonstrate the chiral nature of this overlayer. The homochiral islands typically grow from kinked step edges. While islands of D-cysteine show a strong preference for growing from $S$ kinks (marked by the arrow in Figure 2a), islands of L-cysteine preferentially grow from $R$ kinks (Figure 2b). (In total, 40 such island-kink combinations were observed, compared to $<4$ with the opposite $\mathrm{L}-S$ or $\mathrm{D}-R$ combination.) Thus, a preferential interaction with one type of kink was observed. We speculate that the formation of the dense islands is related to the adsorption of the dimers at the kink sites i.e., with the dimers acting as nucleation sites for the island growth.

In summary, we have presented a direct observation by STM of enantiospecific adsorption of chiral molecules at chiral kink sites. The adsorption geometry at the kinks can be understood from the need to reach specific, optimum molecule-substrate interaction points.

Acknowledgment. This work was supported by the Danish National Research Foundation, the Danish Natural Science Research Council, and the Carlsberg Foundation.

\section{References}

(1) Sheldon, R. A. Chirotechnology 39-72; Dekker: New York/Basel, 1993

(2) Lorenzo, M. O.; Haq, S.; Bertrams, T.; Murray, P.; Raval, R. Nature 2000 404, 376-379.

(3) De Feyter, S.; Grim, P. C. M. Rückert, M.; Vanoppen, P.; Meiners, Ch Sieffert, M.; Valiyaveettil, S.; Müllen, K.; De Schryver, F. C. Angew. Chem., Int. Ed. 1998, 37, 1223-1226.

(4) Fang, H.; Giancarlo, L. C.; Flynn, G. W. J. Phys. Chem. B 1998, 102, $7311-7315$.

(5) Lopinski, G.; Moffatt, D.; Wayner, D.; Wolkow, R. Nature 1998, 392, 909-911.

(6) Eckhardt, C. J.; Peachey, N. M.; Swanson, D. R.; Takacs, J. M.; Khan, M. A.; Gong, X.; Kim, J.-H.; Wang, J.; Uphaus A. Nature 1993, 362, 614-616.

(7) Humbolt, V.; Raval, R. Appl. Surf. Sci. 2005, 241, 150-156.

(8) Weckesser, J.; De Vita, A.; Barth, J. V.; Cai, C.; Kern, K. Phys. Rev. Lett. 2001, 87, 96101

(9) Böhringer, M.; Morgenstern, K.; Schneider, W.-D.; Berndt, R. Angew Chem., Int. Ed. 1999, 38, 821-823.

(10) Kühnle, A.; Linderoth, T. R.; Besenbacher, F. J. Am. Chem. Soc. 2003 $125,14680-14681$.

(11) Kühnle, A.; Linderoth, T. R.; Hammer, B.; Besenbacher, F. Nature 2002, $415,891-893$

(12) Chen, Q.; Richardson, N. V. Nat. Mater. 2003, 2, 324-328.

(13) Zhao, X. J. Am. Chem. Soc. 2000, 122, 12584-12585.

(14) Zhao, X.; Perry, S. S.; Horvarth, J. D.; Gellman, A. J. Surf. Sci. 2004 563, 217-224.

(15) Pascual, J. I.; Barth, J. V.; Ceballos, G.; Trimarchi, G.; De Vita, A.; Kern, K.; Rust, H.-P. J. Chem. Phys. 2004, 120, 11367-11370.

(16) Schunack, M.; Lægsgaard, E.; Stensgaard, I.; Johannsen, I.; Besenbacher, F. Angew. Chem., Int. Ed. 2001, 40, 2623-2626.

(17) Parschau, M.; Romer, S.; Ernst, K.-H. J. Am. Chem. Soc. 2004, 126, $15398-15399$

(18) McFadden, C. F.; Cremer, P. S.; Gellman, A. J. Langmuir 1996, 12, 24832487.

(19) Ahmadi, A.; Attard, G.; Feliu, J.; Rodes, A. Langmuir 1999, 15, 24202424.

(20) Hazen, R. M.; Sholl, D. Nat. Mater. 2003, 2, 367-374.

(21) Pratt, S. J.; Jenkins, S. J.; King, D. A. Surf. Sci. 2005, 585, L159-L165.

(22) Horvarth, J. D.; Koritnik, A.; Kamakoti, P.; Sholl, D. S.; Gellman, A. J. J. Am. Chem. Soc. 2004, 126, 14988-14994.

(23) Sholl, D. S.; Asthagiri, A.; Power, T. D. J. Phys. Chem. B 2001, 105, 4771-4782.

(24) Gellman, A. J.; Horvath, J. D.; Buelow, M. T. J. Mol. Catal. A 2001, 167, 3-11.

(25) Sljivancanin, Z.; Gothelf, K. V.; Hammer, B. J. Am. Chem. Soc. 2002, 124, 14789-14794.

(26) Gritsch, T.; Coulman D.; Behm R. J.; Ertl, G. Surf. Sci. 1991, 257, 297306.

(27) Besenbacher, F. Rep. Prog. Phys. 1996, 59, 1737-1802 (see also http:// www.specs.de).

(28) Kühnle, A.; Molina, L.; Linderoth, T. R.; Hammer, B.; Besenbacher, F. Phys. Rev. Lett. 2004, 93, 86101

JA0568751 